\title{
B Physics at the Tevatron
}

\author{
Karen Gibson*† \\ University of Pittsburgh \\ E-mail: krg20@pitt.edu
}

I present recent results in $B$ physics from the CDF and D0 experiments at the Fermilab Tevatron. A number of $B$ hadron lifetime, spectroscopy, and $C P$ violation results are presented, all of which have been released in 2008. The breadth of the work in $B$ physics that has been produced by the Tevatron experiments indicates the overall success of the program, as well as the new and improved results which can still be anticipated in the final years of Run II.

2008 Physics at LHC

September 29 - 4 October 2008

Split, Croatia

\footnotetext{
*Speaker.

${ }^{\dagger}$ On behalf of the CDF and D0 Collaborations
} 


\section{Introduction}

Over the past five years, the CDF and D0 experiments have produced prolific $B$ physics results during Run II of the Fermilab Tevatron. These results have been complementary in nature to the excellent $B$ physics programs conducted by the BABAR and Belle experiments, both of which operate at electron-positron colliders working at the $\Upsilon(4 S)$ threshold (called $\mathrm{B}$ factories), giving the experiments very clean, high-statistics samples of $B^{0}$ and $B^{+}$mesons[1] to study. ${ }^{1}$ In contrast, the $\sqrt{s}=1.96 \mathrm{TeV} / c^{2} p \bar{p}$ collisions at the Tevatron produce all flavors of $B$ hadrons (in addition to a lot of other things), giving relatively large samples of $B_{s}^{0}$ mesons and $\Lambda_{b}^{0}$ baryons, as well as large samples of light $B^{0}$ and $B^{+}$mesons. This has allowed the Tevatron to measure the frequency of $B_{s}^{0}-\bar{B}_{s}^{0}$ oscillations [2] and discover new $B$ baryons, such as the $\Sigma_{b}^{(*) \pm}[3]$ and the $\Xi_{b}^{-}[4,5]$. With more than $4 \mathrm{fb}^{-1}$ of data on tape, the samples of $B$ hadrons which will be available for analysis will allow (and in some cases already are allowing) $\mathrm{CDF}$ and $\mathrm{D} 0$ to produce $C P$ violation and lifetime measurements in $B^{0}$ and $B^{+}$that compete with the B factories for the most precise results in the world.

In fact, the Tevatron experiments are in a special position; the LHC experiments do not yet have data for $B$ physics, while the B factories have either ceased operation (at SLAC) or will transition within a year from their first run (at KEK) to upgrades which will allow much more integrated luminosity in a few years time. I will highlight recent results from the Tevatron which were made public in 2008 and give some indication of results that can be anticipated when the full Tevatron dataset becomes available. The results presented are divided into three main categories: spectroscopy, lifetimes, and $C P$ violation.

When presenting results from the CDF and D0 experiments, it is worthwhile to note that the two detectors have different strengths in detecting $B$ hadrons. The CDF detector features a strong tracking system that provides good mass and vertex resolution, and the ability to trigger on displaced tracks, which allows CDF to select a large sample of fully-reconstructed $B$ events. The D0 detector has excellent calorimetry and muon identification that provides a large sample of semi-leptonic $B$ decays. The D0 experiment also reverses the direction of the magnetic field in the detector regularly, which is beneficial in direct $C P$ violation measurements by minimizing systematic uncertainties due to charge asymmetries in the data.

\section{Spectroscopy}

In all aspects of particle physics, it is important both to look for things that we expect and also for things that we don't expect. Flavor physics is no exception to this rule and discoveries of the $X$, $Y$, and $Z$ particles by the Belle and BABAR experiments $[6,7]$ demonstrate that unexpected particles are still present in current datasets. Although the $Y$ and $Z$ particles are not studied by the Tevatron experiments, CDF has made several important contributions to the study of the $X(3872)$ particle. The Tevatron experiments have also recently observed some $b$-baryons for the first time, including the $\Sigma_{b}^{ \pm}$baryon, the $\Xi_{b}^{-}$baryon, and, recently, the $\Omega_{b}^{-}$baryon. The existence of these particles has been predicted, but until Run II none had been observed.

\footnotetext{
${ }^{1}$ The Belle experiment has collected some data at the $\Upsilon(5 S)$ threshold, which can decay to $B_{s}^{0}-\bar{B}_{s}^{0}$ pairs, but the sample, which is presently small, has just begun to be used for to produce significant physics results.
} 
The $X(3872) \rightarrow J / \psi \pi^{+} \pi^{-}$was first observed by the Belle collaboration in 2003 [6]. Although five years have passed since its observation, it is still not known whether it is a $D^{*} D$ bound state, a tetra-quark state, a conventional charmonium state, or something else. The CDF collaboration has searched for a mass splitting in order to test the tetra-quark hypothesis, which predicts two states with slightly different masses. If no mass splitting is observed, a precision measurement of the absolute mass is interesting, as it checks whether the mass is within the threshold of a bound-state $D^{*} D$ "molecule" [8].

In order to test the tetra-quark interpretation, the mass distribution of the $X(3872)$ is fit with a Breit-Wigner convolved with detector resolution and a scale factor for the width of the mass distribution. This scale factor is adjusted for different mass-splitting hypotheses in pseudo-experiments to determine whether the two mass hypothesis is consistent with the distribution observed in data. Unfortunately, the data shows no evidence of a mass splitting and an upper limit on the mass splitting is set. The absolute mass is then measured, finding a mass which is below the threshold of a $D^{*} D$ bound state, but which is within $1 \sigma$ of the threshold, leaving open the possibility of the bound-state explanation. The limits on the mass splitting and the absolute mass are

$$
\begin{array}{r}
\Delta m(X(3872))<3.2(3.6) \mathrm{MeV} / c^{2} \text { at } 90 \%(95 \%) \mathrm{CL}, \\
m(X(3872))=3871.61 \pm 0.16 \text { (stat.) } \pm 0.19 \text { (syst.) } \mathrm{MeV} / c^{2} .
\end{array}
$$

One of the most exciting physics results from the Tevatron this year is D0's recent observation of the $\Omega_{b}^{-}$baryon [9] in the decay $\Omega_{b}^{-} \rightarrow J / \psi\left[\rightarrow \mu^{+} \mu^{-}\right] \Omega^{-}\left[\rightarrow \Lambda^{0}\left(\rightarrow p \pi^{-}\right) K^{-}\right]$. This observation was made with $1.3 \mathrm{fb}^{-1}$ of data and builds on D0's previous observation of the $\Xi_{b}^{-} \rightarrow J / \psi[\rightarrow$ $\left.\mu^{+} \mu^{-}\right] \Xi^{-}\left[\rightarrow \Lambda^{0}\left(\rightarrow p \pi^{-}\right) \pi^{-}\right]$[4]. Special selection techniques, such as a boosted decision tree and re-processing the data with a higher impact parameter requirement on tracks to increase acceptance, improved the $\Omega^{-}$reconstruction significantly. The invariant mass distribution of the $\Omega_{b}^{-}$is shown in Fig. 1. D0 observes $17.8 \pm 4.9$ (stat.) \pm 0.8 (syst.) events with $5.4 \sigma$ significance and measures the mass $m\left(\Omega_{b}^{-}\right)=6.165 \pm 0.010$ (stat.) \pm 0.013 (syst.) $\mathrm{GeV} / c^{2}$. D0 measures the production fraction times branching ratio of the observed $\Omega_{b}^{-}$decays relative to the observed $\Xi_{b}^{-}$decay to be

$$
\frac{f\left(b \rightarrow \Omega_{b}^{-}\right) \operatorname{Br}\left(\Omega_{b}^{-} \rightarrow J / \psi / \Omega^{-}\right)}{f\left(b \rightarrow \Xi_{b}^{-}\right) \operatorname{Br}\left(\Xi_{b}^{-} \rightarrow J / \psi / \Xi^{-}\right)}=0.80 \pm 0.32 \text { (stat.) }{ }_{-0.22}^{+0.14} \text { (syst.). }
$$

\section{Lifetimes}

The proper decay times of $B$ hadrons are fundamental quantities, the measurement of which, along with measurements of their masses and decay rates to different final states, helps to paint a complete picture of $B$ hadrons. Precise measurements of $B$ hadron lifetimes test the predictions of the Heavy Quark Effective Theory (HQET), which predicts $\tau\left(B^{+}\right)>\tau\left(B^{0}\right) \approx \tau\left(B_{s}^{0}\right)>\tau\left(\Lambda_{b}^{0}\right)$, all of which are predicted to be significantly longer than the lifetime of the $B_{c}^{+}$. Previously, measurements of $\tau\left(B_{s}^{0}\right)$ and $\tau\left(\Lambda_{b}^{0}\right)$ showed $1-2 \sigma$ discrepancies with the HQET predictions, which were difficult to explain theoretically.

A recent CDF measurement of the $B_{s}^{0}$ lifetime in hadronic $B_{s}^{0} \rightarrow D_{s}^{-}\left[\rightarrow \phi\left(\rightarrow K^{+} K^{-}\right) \pi^{-}\right] \pi^{+}$ decays finds a lifetime of $c \tau\left(B_{s}^{0}\right)=455 \pm 12$ (stat.) \pm 7 (syst.) $\mu \mathrm{m}$ [10], which is compatible 


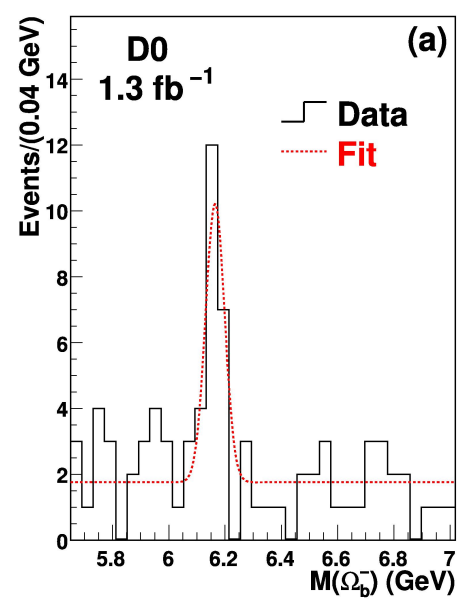

Figure 1: $\Omega_{b}^{-}$invariant mass observed by the D0 collaboration.

with the current average $B^{0}$ lifetime $c \tau\left(B_{s}^{0}\right)=458.7 \pm 2.7 \mu \mathrm{m}$ [11]. A similar measurement of the $\Lambda_{b}^{0}$ lifetime made by CDF in the hadronic decay channel $\Lambda_{b}^{0} \rightarrow \Lambda_{c}^{+}\left[\rightarrow p K^{-} \pi^{+}\right] \pi^{+}$also finds much better compatibility with HQET than had previously been established, with CDF finding $c \tau\left(\Lambda_{b}^{0}\right)=423 \pm 14$ (stat.) \pm 9 (syst.) $\mu \mathrm{m}$ and $c \tau\left(\Lambda_{b}^{0}\right) / c \tau\left(B^{0}\right)=0.92 \pm 0.04$ [12]. Both CDF and D0 collaborations have also measured the $B_{c}^{+}$meson lifetime in semi-leptonic modes. D0 has measured the lifetime in the decay channel $B_{c}^{+} \rightarrow J / \psi \mu^{+} X$, while CDF measures the lifetime in both $B_{c}^{+} \rightarrow$ $J / \psi \mu^{+} X$ and $B_{c}^{+} \rightarrow J / \psi e^{+} X$ decays. The measured lifetimes are consistent between the two experiments, with D0 finding $c \tau\left(B_{c}^{+}\right)=134 \pm 11$ (stat.) \pm 10 (syst.) $\mu \mathrm{m}$ [13] and CDF measuring $c \tau\left(B_{c}^{+}\right)=142 \pm 15$ (stat.) \pm 6 (syst.) $\mu \mathrm{m}$ [14]. All of these recent lifetime measurements are in good agreement with theoretical predictions [15].

\section{CP Violation}

There are three known varieties of $C P$ violation possible in hadron systems. $C P$ violation in the decay of hadrons is called direct $C P$ violation and is the only type of $C P$ violation possible for charged mesons such as the $B^{+}$meson. Indirect $C P$ violation can occur in the mixing of neutral mesons; examples of which include $C P$ asymmetries in semi-leptonic decays of neutral mesons. Finally, $C P$ violation can occur in the interference between mixing and decay. A well-known example of this in $B$ physics is the $C P$-violating phase $\beta$ in the $B^{0} \rightarrow J / \psi K_{s}^{0}$ decay channel, although the analogous $C P$-violating phase $\beta_{s}$, which can be measured in $B_{s}^{0} \rightarrow J / \psi \phi$ decays, has recently generated considerable theoretical and experimental interest. The most powerful measurements of $C P$-violating phases generally involve the use of flavor-tagging, which determines the flavor of the $B$ hadron at production. This task is straight-forward for charged particles like the $B^{+}$, which are self-tagging, but is much more difficult for neutral mesons, such as the $B^{0}$ and $B_{s}^{0}$. Powerful flavor tags include same-side kaon tags and opposite-side lepton and jet-charge tags.

Mixing in neutral meson systems is described by a Schrodinger equation

$$
i \frac{d}{d t}\left(\begin{array}{c}
\left|B_{s}^{0}\right\rangle \\
\left|\bar{B}_{s}^{0}\right\rangle
\end{array}\right)=M^{s}-\frac{i}{2} \Gamma^{s}\left(\begin{array}{c}
\left|B_{s}^{0}\right\rangle \\
\left|\bar{B}_{s}^{0}\right\rangle
\end{array}\right),
$$


where $M^{s}$ is the mass matrix and $\Gamma^{s}$ is the decay matrix that relates the flavor eigenstates $\left|B_{s}^{0}\right\rangle$ and $\left|\bar{B}_{s}^{0}\right\rangle$ to the mass eigenstates $\left|B_{s}^{H}\right\rangle$ and $\left|B_{s}^{L}\right\rangle$, such that

$$
\begin{aligned}
\left|B_{s}^{H}\right\rangle & =p\left|B_{s}^{0}\right\rangle-q\left|\bar{B}_{s}^{0}\right\rangle, \\
\left|B_{s}^{L}\right\rangle & =p\left|B_{s}^{0}\right\rangle+q\left|\bar{B}_{s}^{0}\right\rangle .
\end{aligned}
$$

where $|q / p|=\left|\left(V_{t b} V_{t s}^{*}\right) /\left(V_{t b}^{*} V_{t s}\right)\right| \equiv 1$ is related to the strength of the $t \rightarrow s$ and $t \rightarrow b$ weak transitions described by the CKM quark matrix [16]. The mass difference between the two mass eigenstates gives rise to the oscillation frequency $\Delta m_{s} \equiv m_{H}-m_{L}$ between the $B_{s}^{0}$ and $\bar{B}_{s}^{0}$ and is related to the off-diagonal mass matrix element $\Delta m_{s} \approx 2\left|M_{12}^{s}\right|$, while the width difference $\Delta \Gamma \equiv$ $\Gamma_{L}^{s}-\Gamma_{H}^{s}$ is related to the off-diagonal decay matrix element gives rise to a lifetime difference between the two mass eigenstates $\Delta \Gamma \approx 2\left|\Gamma_{12}^{s}\right| \cos \left(\phi_{s}\right)$, where $\phi_{s}=\arg \left(-M_{12} / \Gamma_{12}\right) \sim 0.004$ in the standard model.

A primary reason for interest in the $C P$-violating phase $\beta_{s}$ in $B_{s}^{0} \rightarrow J / \psi \phi$ decays is that this phase is particularly sensitive to the presence of new physics, since it is theoretically well-predicted from elements of the CKM quark matrix to be nearly zero, $\beta_{s}=\arg \left(-\frac{V_{t s} V_{t b}^{*}}{V_{c s} V_{c b}^{*}}\right) \sim 0.02$ [17]. Consequently, any observation of a large $C P$-violating phase would be an indication of new physics. If present, new physics is likely to appear in the mixing of $B_{s}^{0}$ into $\bar{B}_{s}^{0}$, which involves $t \rightarrow s$ quark transitions in the standard model but which could also involve new, heavy particles.

In late $2007 \mathrm{CDF}$ announced the first flavor-tagged measurement of $\beta_{s}$ in $1.3 \mathrm{fb}^{-1}$ of data and observed a deviation from the standard model prediction with a $1.5 \sigma$ significance [18]. CDF updated this result for the summer of 2008 , using data through $2.8 \mathrm{fb}^{-1}$, and finds that the discrepancy remains and its significance increases to $1.8 \sigma$ [19]. D0 finds a completely compatible flavor-tagged result in $2.8 \mathrm{fb}^{-1}$ of data, observing a discrepancy with the standard model of $1.7 \sigma$ [20]. A recent combination of the original $1.3 \mathrm{fb}^{-1} \mathrm{CDF}$ result and the D0 result by the Heavy Flavor Averaging Group (HFAG), shown in Fig. 2, finds a discrepancy with the standard model of $2.2 \sigma$. While not statistically significant, this result is very intriguing, particularly when taken in conjunction with the asymmetry of direct $C P$ asymmetries in $B^{0}$ and $B^{+}$decays to $K \pi$ final states observed by the Belle and BABAR collaborations [21]. George Hou et al. have predicted that both sets of discrepancies could be due to the presence of a heavy $t^{\prime}$ quark, with a mass between $300 \mathrm{GeV} / c^{2}$ and $\sim 1 \mathrm{TeV} / c^{2}$ [22], as both effects involve $t \rightarrow s$ quark transitions.

The D0 collaboration has also recently announced a time-dependent, flavor-tagged measurement of the semi-leptonic $B_{s}^{0}$ asymmetry $a_{s l}^{s}$, measured in the decay $B_{s}^{0} \rightarrow D_{s}^{-}\left[\rightarrow \phi \pi^{-}\right] \mu^{+} v_{\mu} X$ [23]. The time dependence of this decay depends on $\tau\left(B_{s}^{0}\right), \Delta \Gamma, \Delta m_{s}$. D0 performs a maximum likelihood fit to extract $a_{s l}^{s}=-0.0024 \pm 0.0117$ (stat.) ${ }_{-0.0024}^{+0.0015}$ (syst.), which is the most precise measurement of this quantity to date.

Another probe of the mixing-related quantities in the $B_{s}^{0}$ system is the measurement of the branching ratio of $B_{s}^{0} \rightarrow D_{s}^{(*)+} D_{s}^{(*)-}$ to determine $\Delta \Gamma$. D0 has measured this branching fraction by fully reconstructing one $D_{s}$ in the $\phi\left[\rightarrow K^{+} K^{-}\right] \pi$ decay mode and the other $D_{s}$ semi-leptonically in the $\phi \mu v_{\mu} X$ decay mode [24]. Under certain theoretical assumptions [17], the $B_{s}^{0} \rightarrow D_{s}^{(*)+} D_{s}^{(*)-}$ decay is nearly $C P$ even, giving

$$
2 B r\left(B_{s}^{0} \rightarrow D_{s}^{(*)+} D_{s}^{(*)-}\right) \approx \Delta \Gamma^{C P}\left[\frac{1+\cos \phi_{s}}{2 \Gamma_{L}}+\frac{1-\cos \phi_{s}}{2 \Gamma_{H}}\right] .
$$




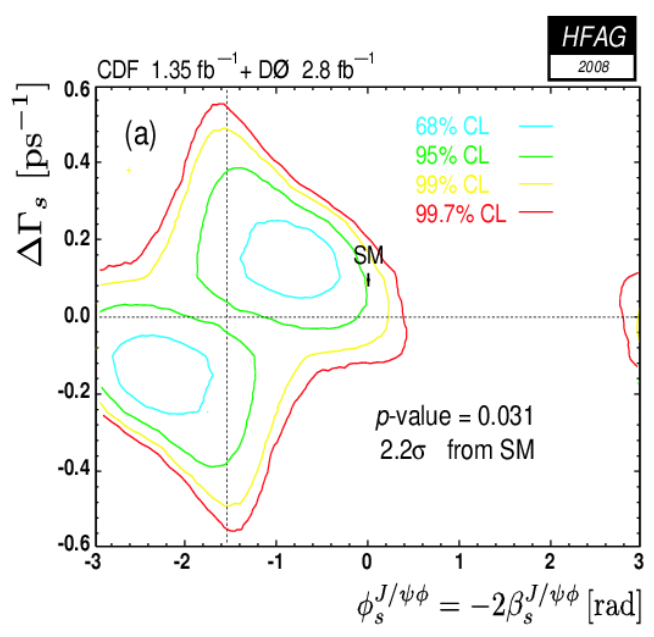

Figure 2: HFAG combination of CDF and D0 results in $\phi_{s}^{J / \psi \phi}-\Delta \Gamma$ plane. Note that $\phi_{s}^{J / \psi \phi}-\Delta \Gamma \equiv-2 \beta_{s}$.

D0 finds a branching ratio $\operatorname{Br}\left(B_{s}^{0} \rightarrow D_{s}^{(*)+} D_{s}^{(*)-}\right)=0.042 \pm 0.015$ (stat.) \pm 0.017 (syst.). Under standard model assumptions $\phi_{s}=0.004 \approx 0$ and $\Delta \Gamma^{C P}=\Delta \Gamma$. D0 extracts $\Delta \Gamma / \Gamma=0.088 \pm$ 0.030 (stat.) \pm 0.017 (syst.), which is consistent with the HFAG average $\Delta \Gamma / \Gamma=0.096_{-0.053}^{+0.048}$ [25].

Finally, D0 recently measured the direct $C P$ asymmetry in $B^{+} \rightarrow J / \psi K^{+}\left(\pi^{+}\right)$decays [26]. The standard model predicts that $A_{C P}\left(B^{+} \rightarrow J / \psi K^{+}\right) \sim 0.003$, although new physics may give rise to asymmetries as large $\sim 0.01$. Clearly this is a measurement which requires high precision in order to test the standard model. The $C P$ asymmetry measured is defined as

$$
A_{C P}\left(B^{+} \rightarrow J / \psi K^{+}\left(\pi^{+}\right)\right)=\frac{N\left(B^{-} \rightarrow J / \psi K^{-}\left(\pi^{-}\right)\right)-N\left(B^{+} \rightarrow J / \psi K^{+}\left(\pi^{+}\right)\right)}{N\left(B^{-} \rightarrow J / \psi K^{-}\left(\pi^{-}\right)\right)+N\left(B^{+} \rightarrow J / \psi K^{+}\left(\pi^{+}\right)\right)}
$$

D0 obtains $A_{C P}\left(B^{+} \rightarrow J / \psi K^{+}\right)=+0.007 \pm 0.0061$ (stat.) \pm 0.0027 (syst.), which is approaching the sensitivity necessary for test of the standard model, and $A_{C P}\left(B^{+} \rightarrow J / \psi \pi^{+}\right)=-0.09 \pm$ 0.08 (stat.) \pm 0.03 (syst.).

\section{Outlook}

Looking beyond the present set of $B$ physics results from the Tevatron, a number of new and updated measurements can be anticipated. Most of the Tevatron $C P$ violation measurements will be updated with two to four times the data presently used by the end of Run II. In addition, now that the flavor-tagging is well-established and statistics are increasing, more tagged $C P$ violation measurements can be expected. The lifetime measurements can be updated with higher statistics; updated $B \rightarrow J / \psi X$ lifetime measurements are expected with twice the statistics of previous measurements, which should give the most precise $B^{+}$and $\Lambda_{b}^{0}$ lifetimes to date. Finally, while never something to be counted on, it is reasonable that the Tevatron may still hold a surprise or two concerning the observation of new states or new physics in the $B_{s}^{0}$ mixing phase. This is an exciting period for Tevatron $B$ physics, with many significant contributions made already and many more to come within the next couple of years. 


\section{References}

[1] Charge-conjugate states are implied throughout the paper unless otherwise specified.

[2] A. Abulencia et al. (CDF Collaboration), Phys. Rev. Lett. 97,242003 (2006).

[3] T. Aaltonen et al. (CDF Collaboration), Phys. Rev. Lett. 99, 202001 (2007).

[4] V. M. Abazov et al. (D0 Collaboration), Phys. Rev. Lett. 99, 052001 (2007).

[5] T. Aaltonen et al. (CDF Collaboration), Phys. Rev. Lett. 99, 052002 (2007).

[6] S. K. Choi et al. (Belle Collaboration), Phys. Rev. Lett. 91, 262001 (2003).

[7] P. Pakhlov et al. (Belle Collaboration), Phys. Rev. Lett. 100, 202001 (2008); K. Abe et al. (Belle Collaboration), Phys. Rev. Lett. 94, 182002 (2005); B. Aubert et al. (BABAR Collaboration), Phys. Rev. Lett.95, 142001 (2005).

[8] http://www-cdf.fnal.gov/physics/new/bottom/080724.blessed-X-Mass/cdfpubnote.ps

[9] V. M. Abazov et al. (D0 Collaboration), arXiv:0808.4142 [hep-ex] (2008).

[10] http://www-cdf.fnal.gov/physics/new/bottom/080207.blessed-bs-lifetime/pubnote_v2.ps

[11] C. Amsler et al., Physics Letters B 667, 1 (2008).

[12] http://www-cdf.fnal.gov/physics/new/bottom/080703.blessed-lblcpi-ct/lambdab_lifetime_public.pdf

[13] V. M. Abazov et al. (D0 Collaboration), arXiv:0805.2614 [hep-ex] (2008).

[14] http://www-cdf.fnal.gov/physics/new/bottom/080327.blessed-BC_LT_SemiLeptonic/blessed-bclifetime.pdf

[15] V. V. Kiselev, hep-ph/0308214 (2003).

[16] N. Cabibbo, Phys. Rev. Lett. 10, 531 (1963);

M. Kobayashi and T. Maskawa, Prog. Theor. Phys. 49, 652 (1973).

[17] I. Dunietz, R. Fleischer, and U. Nierste, Phys. Rev. D 63, 114015 (2001).

[18] T. Aaltonen et al. (CDF Collaboration), Phys. Rev. Lett. 100, 161802 (2008).

[19] http://www-cdf.fnal.gov/physics/new/bottom/080724.blessedtagged_BsJPsiPhi_update_prelim/public_note.pdf

[20] V. M. Abazov et al. (D0 Collaboration), arXiv:0802.2255 [hep-ex] (2008).

[21] The Belle Collaboration, Nature, 452, 332 (2008);

B. Aubert et al. (BABAR Collaboration), Phys. Rev. Lett. 99, 021603 (2007);

B. Aubert et al. (BABAR Collaboration), Phys. Rev. D 76, 091102 (2007).

[22] W.-S. Hou, M. Nagashima, and A. Soddu, Phys. Rev. Lett. 95, 141601 (2005); W.-S. Hou, M. Nagashima, and A. Soddu, Phys. Rev. D 76, 016004 (2007).

[23] http://www-d0.fnal.gov/Run2Physics/WWW/results/prelim/B/B55/B55.pdf

[24] V. M. Abazov et al. (D0 Collaboration), arXiv:0811.2173 [hep-ex] (2008).

[25] E. Barberio et al., arXiv:0808.1297 and online update at http://www.slac.stanford.edu/xorg/hfag.

[26] V. M. Abazov et al. (D0 Collaboration), Phys. Rev. Lett. 100, 211802 (2008). 